Rapid Reviews COVID-19•

\title{
Reviews of "Anti-PF4 levels of patients with VITT do not reduce 4 months \\ following AZD1222 vaccination"
}

Kochawan Boonyawat ${ }^{1}$, Helmuth Haslacher ${ }^{2}$, Thomas Perkmann², Pier Meroni ${ }^{3}$, Maria Orietta Borghi ${ }^{3}$

${ }^{1}$ Mahidol University, ${ }^{2}$ Medical University of Vienna, ${ }^{3}$ Fondazione Istituto Auxologico Italiano

Published on: Sep 14, 2021

DOI: 10.1162/2e3983f5.9198d3b2

License: Creative Commons Attribution 4.0 International License (CC-BY 4.0). 
To read the original manuscript, click the link above.

Summary of Reviews: This paper claims that, although anti-PF4 antibody levels remain high in VITT patients months after follow-up, it is not associated with increased platelet activation Reviewers found it timely and reliable but in need of minor revisions on its methodology and discussion.

\section{Reviewer 1 (Kochawan Boonyawat) |}

Reviewer 2 (Helmuth Haslacher, Thomas Perkmann) |

\section{Reviewer 3 (Pier Meroni, Maria Orietta Borghi) |}

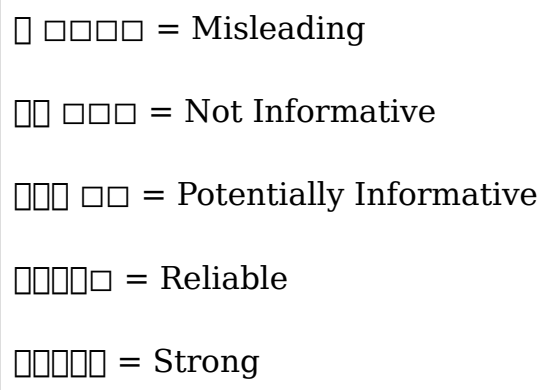

To read the reviews, click the links below. 